\title{
DEVELOPMENT AND AREA-WIDE APPLICATION OF BIOLOGICAL CONTROL USING THE PARASITOID Aphidius gifuensis AGAINST Myzus persicae IN CHINA
}

\author{
Y. B. YU ${ }^{1}$, H. L. YANG 2 , Z. $\mathrm{LIN}^{1}$, S. Y. YANG ${ }^{2}$, L. M. ZHANG ${ }^{2}$, X. \\ H. $\mathrm{GU}^{2}, \mathrm{C} . \mathrm{M} . \mathrm{LI}^{3}$ AND X. WANG ${ }^{4}$ \\ ${ }^{1}$ Yunnan Tobacco Company of China National Tobacco Corporation (CNTC), \\ Kunming, Yunnan 650011, China; yuyanbi@sina.com \\ ${ }^{2}$ Yuxi Branch of Yunnan Tobacco Company of CNTC, Yuxi, Yunnan, 653100, China \\ ${ }^{3}$ Honghe Branch of Yunnan Tobacco Company of CNTC, Honghe, Yunnnan, \\ 652300,China; 3641775@qq.com \\ ${ }^{4}$ Dali Branch of Yunnan Tobacco Company of CNTC, Dali, Yunnan, 671000, China
}

\begin{abstract}
SUMMARY
Ecologically safe and environment-friendly pest control strategies and technologies are important to ensure the quality of Chinese agricultural products and sustainable agricultural development. Aphids are among the world's major agricultural and forest pests, and Myzus persicae Sulzer (Hemiptera: Aphididae) is one of the main agricultural pests in China, transmitting various viral diseases and causing reductions in crop yield and quality that regularly triggered applications of synthetic insecticides. Aphidius gifuensis Ashmead (Hymenoptera: Braconidae) is an important endoparasitoid of many aphids. Starting in 2000, the Yunnan Tobacco Company has developed methods for large-scale rearing of this parasitoid on this aphid, and technological systems for augmentative releases of $A$. gifuensis. The augmentative use of this parasitoid has achieved area-wide suppression of M. persicae in tobacco and other crops in China. This approach is being applied on large areas, covering more than 3 million ha between 2010 and 2015. This programme is currently the largest biological control programme in China. Over 500 mass-rearing facilities were constructed in 16 provinces with a total surface area of $420000 \mathrm{~m}^{2}$ and a breeding capacity of 24000 million parasitoids per breeding period. This technology has effectively controlled the aphid on tobacco, while other beneficial insects have increased in the absence of insecticide applications, further protecting biodiversity in the fields and providing long-term ecological benefits. The use of this technology has also been expanded to other crops, solving problems of insecticide resistance in the targeted aphids, reducing pesticide residues and environmental pollution, and yielding benefits for society, the economy, and the environment.
\end{abstract} Development and Field Application, pp. 3-16. CRC Press, Boca Raton, Florida, USA. 
Key Words: Aphididae, aphids, augmentative biological control, tobacco, technology research, technology transfer, large-scale parasitoid breeding, Braconidae, release technique, training, extension, environmentfriendly pest control, Yunnan

\section{INTRODUCTION}

Yunnan Province, China is one of the most important tobacco growing regions in the world with 469000 ha under cultivation, representing 35\% and 20\% of tobacco production in China and the world, respectively. Yunnan province is also famous for producing tobacco of high quality, and its tobacco industry has provided a sustainable livelihood and alleviated poverty for more than 800000 farming families. Tobacco in Yunnan province is usually planted in mountainous areas, of high scenic value, that have fragile ecosystems. The Yunnan tobacco-planting region has five characteristics: (1) a wide distribution of planting sites, (2) planting in a variety of ecological regions, (3) dominance by smallholder farmers, (4) farmers of widely different backgrounds, and (5) a mosaic of factors influencing tobacco leaf yields and quality.

Aphids are among the most destructive pests, are often highly polyphagous and impact a broad range of agricultural crops worldwide. Plant sap-sucking and honeydew production by the tobacco aphid Myzus persicae Sulzer (Hemiptera: Aphididae) directly injures the host plant, causing significant yield reduction (Kulash 1949; Starý 1970). In addition, damage from M. persicae is exacerbated by its ability to transmit over 100 viral diseases to more than 400 host plants (Mackauer and Way 1976). Most of these viral diseases cause a decline in tobacco yield and quality.

In Yunnan, control of this aphid pest has largely been dependent on insecticides (Zhao et al. 1980), leading to problems of resistance, difficulty of control, destruction of natural enemies, decrease of biodiversity, excessive pesticide residues, reduction in product quality due to repeated applications, inappropriate application methods and incorrect application rates (Gao et al. 1992; Han et al. 1989; He 2013).

In view of these problems, there was a strong need to develop and apply biological control tactics to replace the chemical control systems applied against M. persicae, taking into account the population dynamics of the pest, herbivore-natural enemy interactions, and the economic relationship between pest infestation and crop yield loss (Guan et al. 2016).

Aphidius gifuensis Ashmead (Hymenoptera: Braconidae) is one of the most important natural enemies of aphids and is found in many habitats (Chen 1979). This natural enemy is widely distributed all over the world, for example Canada, China, India, Japan, and USA, where a good basis of ecological knowledge has been accumulated for wider application. Also, many biological and ecological studies of this insect have been conducted in China (Bi and Ji 1993; Lu et al. 1993; Lu et al. 1994).

After mating, females of $A$. gifuensis search for hosts and lay their eggs inside aphid bodies. The eggs develop by absorbing the nutrients from the aphid, and the development of the parasitoid results in the death of the aphid (Ohta et al. 2001) (Fig. 1). 


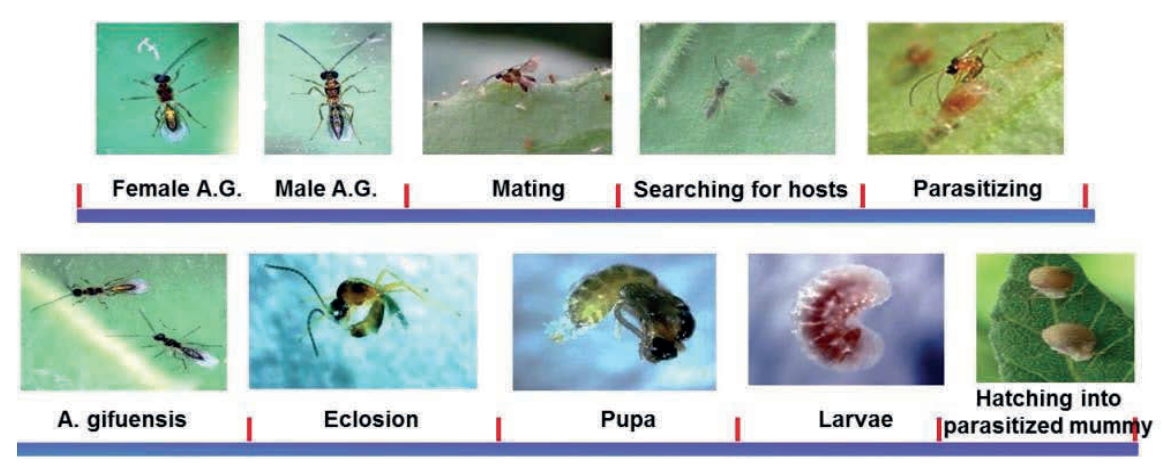

Figure 1. Life cycle and biological stages of Aphidius gifuensis (A.G.) (starting clockwise from top left).

There are two main challenges with respect to the biological control of aphids by using A. gifuensis on a large scale. The first is to develop methods for the large-scale production of the parasitoid and its release. The second is to find mechanisms to transfer this technology to technicians and farmers so that these smallholders can benefit from the technology.

Therefore, after carrying out the research, we have developed and established two systems for effective, economic, and convenient high-density mass-rearing technologies for different application areas, named adult-plant breeding and seedling breeding, published an industry-level standard, constructed a "one plus two" model of technology extension, and achieved area-wide application of the technology for sustainable aphid control by $A$. gifuensis.

\section{MASS-REARING SYSTEMS}

The large-scale mass-rearing of $A$. gifuensis is mainly divided into three parts: (1) cultivating of host plants for $M$. persicae, (2) breeding large populations of $M$. persicae, and (3) high-density mass-rearing of A. gifuensis on M. persicae (Gu et al. 2015). The following summarizes the optimal conditions and procedures for these technologies (Deng et al. 2010, 2011).

\subsection{Adult-Plant Breeding System}

\subsubsection{Cultivating Host Plants}

Tobacco plants that are highly resistant to tobacco mosaic virus (TMV) were selected for mass-rearing of the aphids, i.e. tobacco variety Yunyan 203, as well as white radish and Chinese cabbage variety Chinese 82 . The host plants are seeded and transplanted after 70 to 80 days and grown for 25-30 days till the 6-8 leaves stage for further use. 
2.1.2. Mass-rearing of Myzus persicae

Each tobacco plant, at the 6-8 leaves stage, is inoculated with 20 healthy aphids (nymphs and adults), followed by rearing of the aphids for $15-20$ days at $17-27^{\circ} \mathrm{C}$ and $50-80 \%$ RH in a greenhouse (50 m x $12 \mathrm{~m}$ x $4.6 \mathrm{~m}$ ) (Wu et al. 2000; Deng et al. 2006; Yang and Zhao 2009; Yang et al. 2009) (Fig. 2).

\subsubsection{High-density Mass-rearing of A. gifuensis on Aphids}

When the tobacco leaves are incubated with aphids for 15-20 days, A. gifuensis are released at a parasitoid to aphid ratio of between 1:50 and 1:100. After A. gifuensis females have laid their eggs, the parasitized aphids form mummies, from which a new generation of $A$. gifuensis emerges. After 10 to 15 days, parasitism rates of $>90 \%$ are obtained (Wei et al. 2003, 2005; Yang et al. 2009).

Each tobacco plant can produce $6000-10000$ A. gifuensis adults, and each small greenhouse ( $3 \mathrm{~m} \times 3 \mathrm{~m} \times 2 \mathrm{~m}$ ) containing 28 plants, can produce $160800 \mathrm{~A}$. gifuensis individuals (Fig. 2).

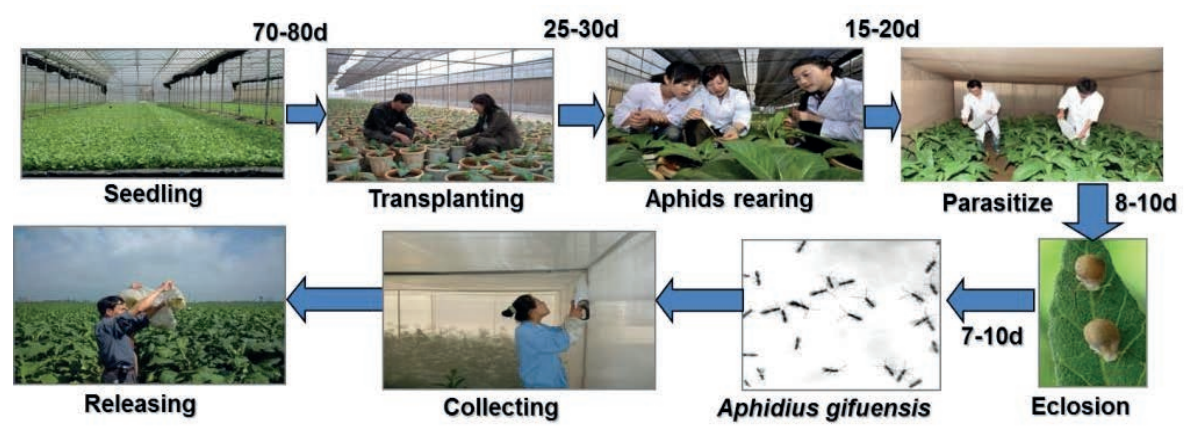

Figure 2. Breeding process of Aphidus gifuensis in greenhouses (starting clockwise from top left).

\subsection{Seedling Breeding System}

\subsubsection{Cultivating Host Plants}

Tobacco variety Yunyan 203 with high resistance to TMV is bred according to the China National Standard GB / 25241 (Liu et al. 2010). Tobacco seedlings with 5 leaves and 1 heart can be used to breed aphids by the "Method of breeding aphid and Aphidus gifuensis separately".

Alternatively, tobacco seedlings in the 3rd - 4th leaf stage can be used to breed aphids by the "Method of breeding aphid and Aphidus gifuensis at the same time" as described below. 


\subsubsection{Rearing of Large Populations of Myzus persicae}

Mass-rearing of aphids is done using two alternative methods:

Breeding aphid and A. gifuensis separately: On tobacco plants with 5 leaves and 1 heart, leaves are inoculated at a rate of 10 aphids per plant. After 10 to 12 days, at 20 to $30^{\circ} \mathrm{C}$ and $60-80 \% \mathrm{RH}$, when the aphid density reaches 200 per plant on average, the population of aphids is ready for use for rearing natural enemies (Fig. 2).

Breeding aphid and A. gifuensis at the same time: On tobacco plants in the 3rd 4th leaf stage, leaves are inoculated with 2.5 aphids per plant (aphids with a parasitism rate from $40 \%$ to $60 \%$ are used for the inoculation, or a parasitoid-aphid ratio of between 1:20 and 1:10), at $20-30^{\circ} \mathrm{C}$ and $60-80 \% \mathrm{RH}$.

\subsubsection{High-density Breeding of A. gifuensis on Aphids}

Parasitoid mass-rearing on aphids is done following two alternative methods:

Breeding aphid and A. gifuensis separately: According to the population of aphids per single plant, A. gifuensis or parasitised aphids are inoculated onto leaves in the greenhouse. After 17 days, the population of parasitised aphids will reach one 100000 per square meter (Fig. 2).

Breeding aphid and A. gifuensis at the same time: A. gifuensis parasitoids are inoculated onto leaves at the same time as aphids. After the A. gifuensis parasitoids emerge from the parasitised aphids, they will parasitize other aphids. If the parasitism rate is too high, more aphids need to be added; alternatively, if the parasitism rate is too low, more A. gifuensis will need to be added. After 23 days, the population of parasitised aphids will reach 49000 per square meter (Fig. 3) (Chen et al. 2009).

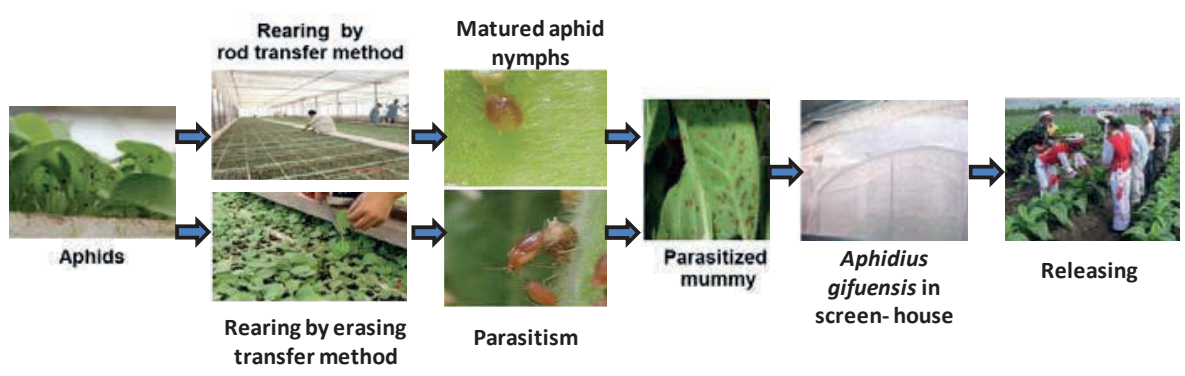

Figure 3. Breeding process of aphids and Aphidius gifuensis.

\subsection{Collection and Storage of $\mathrm{A}$. gifuensis}

There are two methods for collection of $A$. gifuensis:

Manual collection: Home-made, simple collection devices or automatic collection systems (aspirators) are used to collect A. gifuensis in the tents, and A. gifuensis are stored in containers (Fig. 4). 
Automatic collection: Collection bags are placed in the breeding screen-houses (nylon-net covered cages or tents) in tobacco fields, and A. gifuensis adults will fly into the bags as a result of their phototaxis (Fig. 4).

\subsection{Release of $\mathrm{A}$. gifuensis}

Different methods are used to release $A$. gifuensis:

1. Release of parasitised aphids

2. Release of $A$. gifuensis, and

3. Self-dispersal in the field.

When releasing parasitised aphids, leaves or seedling with parasitised aphids are brought to the field and hung onto plants.

When releasing $A$. gifuensis, parasitoids are taken to the field in collection bags or bottles and released before 12 o'clock in the absence of any rain; total transport time should be less than three hours.

For self-dispersal of parasitoids in the field, they are bred in the screen-houses or breeding tents in the field. The tents are opened when parasitism reaches $90 \%$, and the A. gifuensis will disperse naturally to find aphids (Fig. 4).

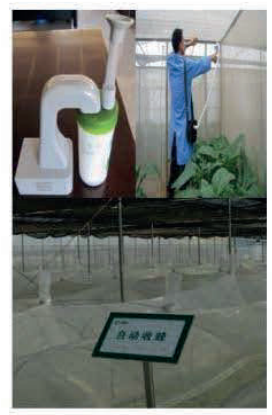

Collection of A. gifuensis

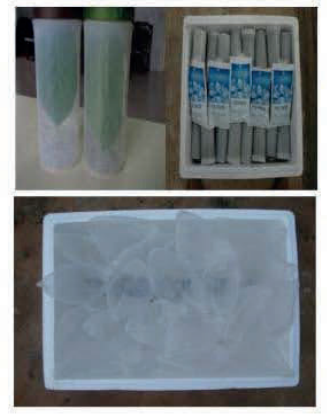

Storage of A. gifuensis

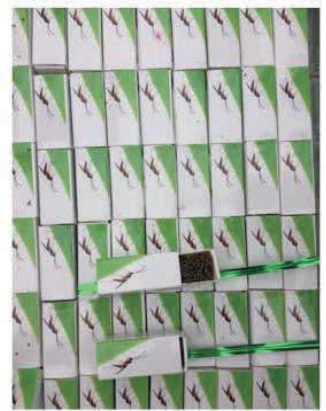

Product of A. gifuensis (Boxes of parasitized aphids)

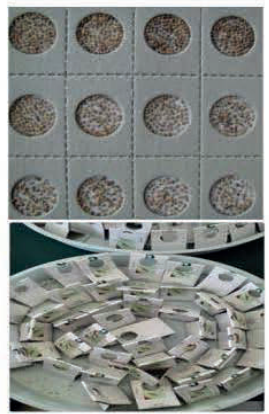

Product of A. gifuensis (Cards of parasitized aphids)

Figure 4. Collection and release processes for Aphidius gifuensis parasitoids.

The density of aphids needs to be assessed to determine the parasitoid release time and numbers to be released. When the population of aphids per plant reaches an average of 1 to 5 individuals, A. gifuensis parasitoids are released at a rate of 3000 to 7500 per ha. The subsequent second and third release are adjusted according to aphid densities.

When the population of aphids per plant reaches an average of 6 to 20, A. gifuensis parasitoids are released at a rate of 7500 to 15000 per ha. When the population of aphids per plant is more than 20, A. gifuensis parasitoids are released at a rate of 15 000-18 000 per ha. 


\subsection{Conservation of Aphid and A. gifuensis Colonies in Winter}

\subsubsection{Conservation of Aphids in Winter}

The main method to maintain a colony of aphids in the winter is by conserving the host plants in greenhouses. Aphids are collected from the wild and inoculated onto healthy tobacco seedlings, cabbage, radish or other host plants. The breeding conditions are held at temperatures between 17 and $27^{\circ} \mathrm{C}$ and 50 and $80 \% \mathrm{RH}$. The status of aphids and host plants is observed, and old and diseased aphids and host plants infected with virus are removed at three different times. Healthy aphids are obtained and used to reinvigorate the colony.

\subsubsection{Conservation of A. gifuensis in Winter}

Holding-over host plants with aphids in the greenhouse or cold storage of parasitised aphids are the two methods used for storing A. gifuensis in winter. A. gifuensis are collected from the wild and used to parasitize aphids several times. To obtain healthy A. gifuensis, the colony needs to be maintained at a temperature of $17-27^{\circ} \mathrm{C}$ and 50 $80 \% \mathrm{RH}$.

When in cold storage, parasitised aphids are collected using a brush or other tools to directly collect them from host plants, placing aphids into tubes, which are then held at $4-5^{\circ} \mathrm{C}$. The seedlings or larger plants with parasitised aphids can also directly be placed into $4-5^{\circ} \mathrm{C}$. Parasitoid emergence rates of up to $90 \%$ are obtained after cold storage for 20 days.

\section{TECHNOLOGY TRANSFER AND APPLICATION SYSTEMS}

\subsection{Technical Standard}

An industrial standard named "Technical specification for Myzus persicae biological control with Aphidius gifuensis (YC/T 437-2012)" was published (Yun et al. 2012), including conservation, colony rejuvenation, large-scale breeding, collection and release.

A total of 506 breeding facilities were built in 16 provinces (regions, cities) in China, with a total surface area of $420000 \mathrm{~m}^{2}$ and a breeding capacity of 24000 million parasitoids per breeding period.

\subsection{Training}

To spread and transfer this technology, a training system was developed and implemented on four levels, i.e. industrial, provincial, municipal and county level. The training programme was developed based on research conducted by the tobacco industry, and also based on field experience by extension services of the government (Fig. 5). 


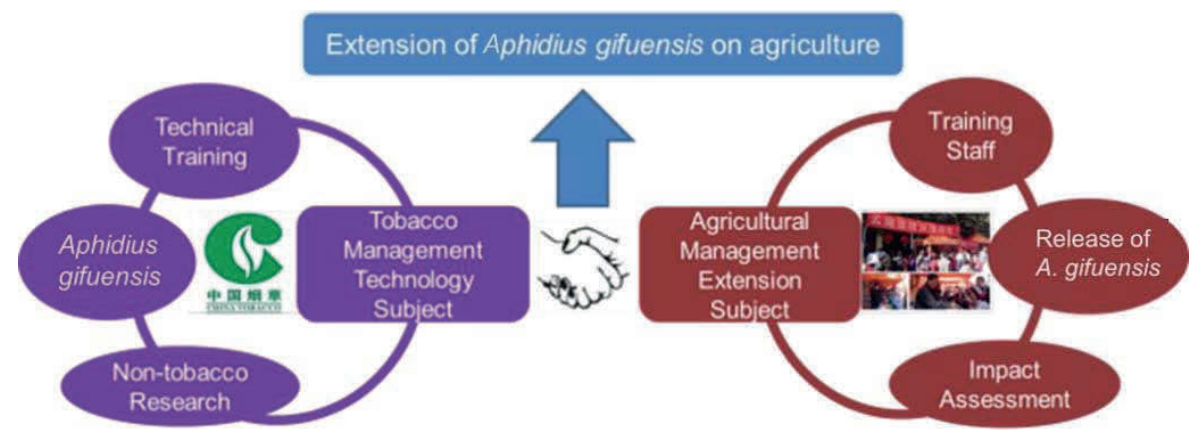

Figure 5. Extension system for biological control of tobacco aphid by parasitoid augmentative releases.

We developed the training platform, carried out theoretical and practical training for the technical experts, technicians, extension workers and farmers, covering stepby-step the key points and difficulties for technicians during programme operation (Fig. 6).

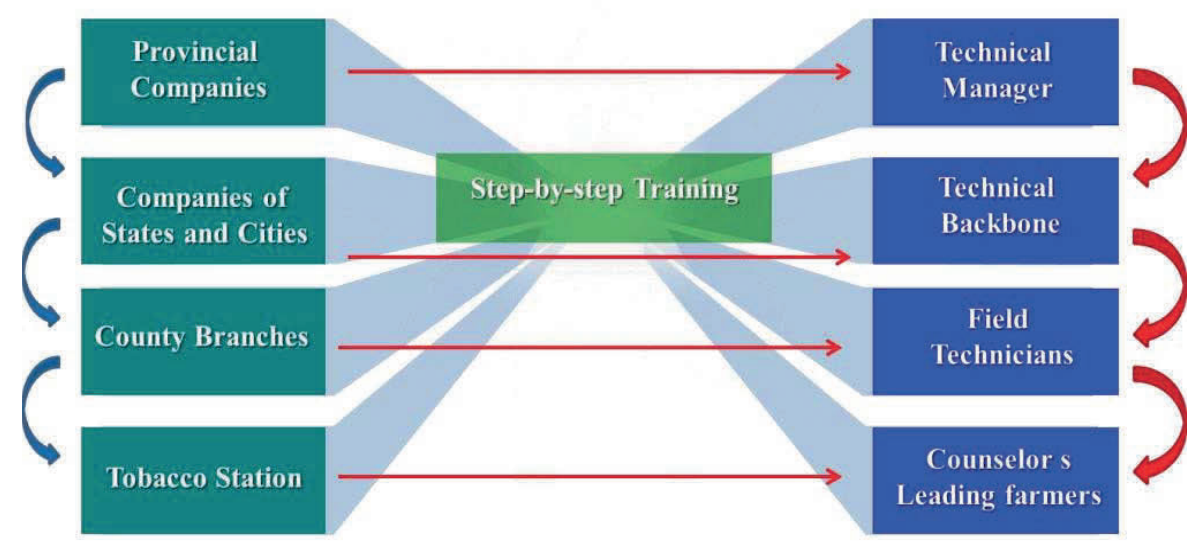

Figure 6. Training system for biological control of tobacco aphid Aphidius gifuensis by parasitoids.

Overall, we have trained more than 3000 core experts and more than 20000 technicians, and also provided more than 1200 thousand copies of technical training books and booklets (Fig. 7). More than 120000 farms benefited from this training and extension work at all levels (Fig. 8). 


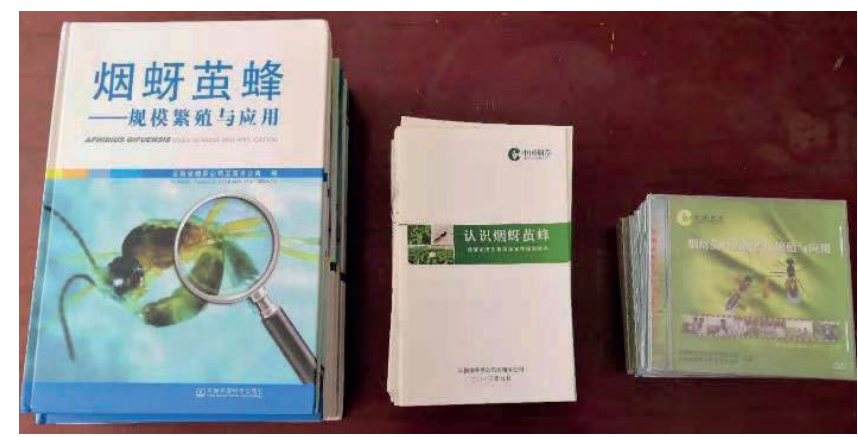

Figure 7. Technical materials on the use of Aphidius gifuensis parasitoids.

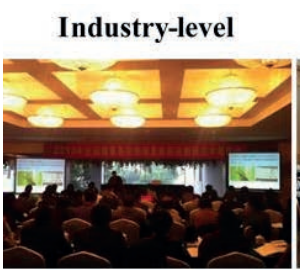

Province-level
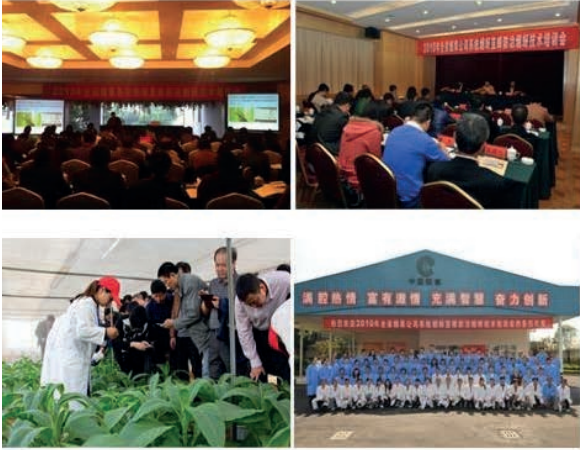

City-level
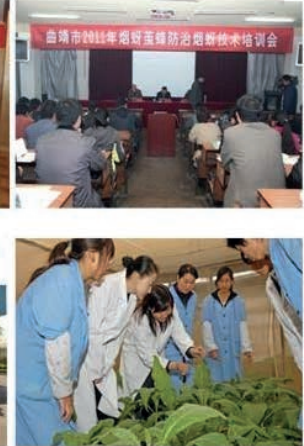

\section{County-level}
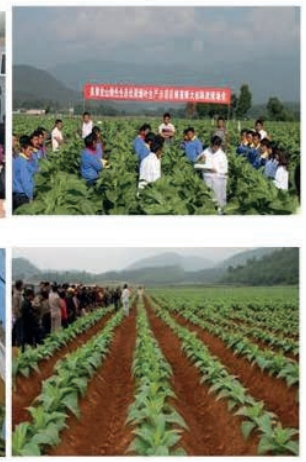

Figure 8. Four-level training for biological control of tobacco aphid by mass-release of Aphidius gifuensis parasitoids.

\subsection{Goal Setting}

Depending on the densities of the aphids in the farms with tobacco fields, A. gifuensis are released following five release densities, i.e. 7500, $12000,15000,18000$ and 22500 parasitoids per ha. Also, depending on the aphid infestation pressure in different areas, application rates are $30 \%, 50 \%, 80 \%$ and $95 \%$ of the total tobaccoplanting area.

\subsection{Matching of Funding}

The funding for the technical application is contained within technical project funds. Each provincial tobacco company matches the obtained funding of USD 11.3-22.6 per ha according to set application areas. 


\subsection{Evaluation}

The programme evaluation is carried out to check implementation, supply, and application scale, and to control efficiency of the application of this technology at different levels. To promote the tobacco aphid biocontrol technique, a series of rewards and penalties were established, with the outcomes of the evaluation results directly tied to the salary of technicians and also to the funding for application in the next year.

\section{IMPACT ASSESSMENT}

Each small greenhouse containing 28 plants can produce ca. 17000 A. gifuensis individuals for release that can protect six ha of tobacco plants. Compared to chemical control of aphids, the cost of aphid control by A. gifuensis mass-releases is much lower. The cost of biological control is estimated at about USD 13.3 per ha, compared to USD 244.8 per ha for the chemical control applying insecticides. Table 1 compares the sub-item costs for both treatments.

Table 1. Comparison of costs of biological and chemical control of aphids

\begin{tabular}{lclc}
\hline \multirow{2}{*}{ Treatments } & \multicolumn{1}{c}{ Total Cost (USD/ha) } & \multicolumn{1}{c}{ Sub-items } & Sub-items costs (USD $/$ ha) \\
\hline Insecticide & 244.8 & Cost of insecticides & $13.6 \times 3$ times $=40.8$ \\
& & Cost of labour & $68.0 \times 3$ times $=204.0$ \\
Biocontrol & 13.3 & Cost of facilities & 3.8 \\
& & Cost of mass-rearing & 5.0 \\
& & Cost of releasing & 4.5 \\
\hline
\end{tabular}

In addition, aphids were well controlled because of the sustained, long-term release of A. gifuensis (Yang et al. 2010) (Fig. 9 and 10).

Meanwhile, populations of other beneficial insects, such as the predators Coccinella septempunctata (L.), Harmonia axyridis (Pallas), Episyrphus balteatus (De Geer), Chrysopa sinica (Tjeder), and Lycosa pseudoamulata Boes. et Str. obviously increased in the absence of insecticide applications, further protecting biodiversity in the fields and providing long-term ecological benefits resulting from the biological control (Shen et al. 2018).

Application of this technology was started in 2000 in the tobacco-planting area of Yuxi, Yunnan Province (Yunnan Tobacco Yuxi City Company 2010), and by 2010 the entire tobacco planting area of Yuxi had been covered (AERET 2011; Yang et al. 2011). 


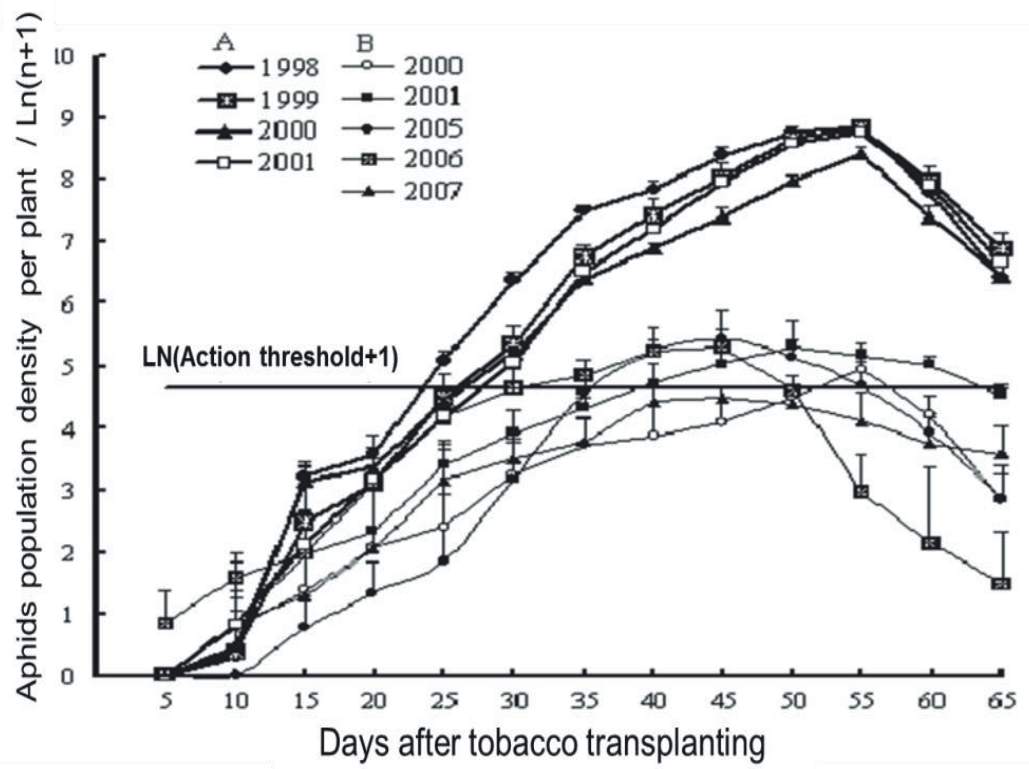

Figure 9. Aphid population control results without A. gifuensis between 1998-2001 (A) and with A. gifuensis between 2000-2007 (B).

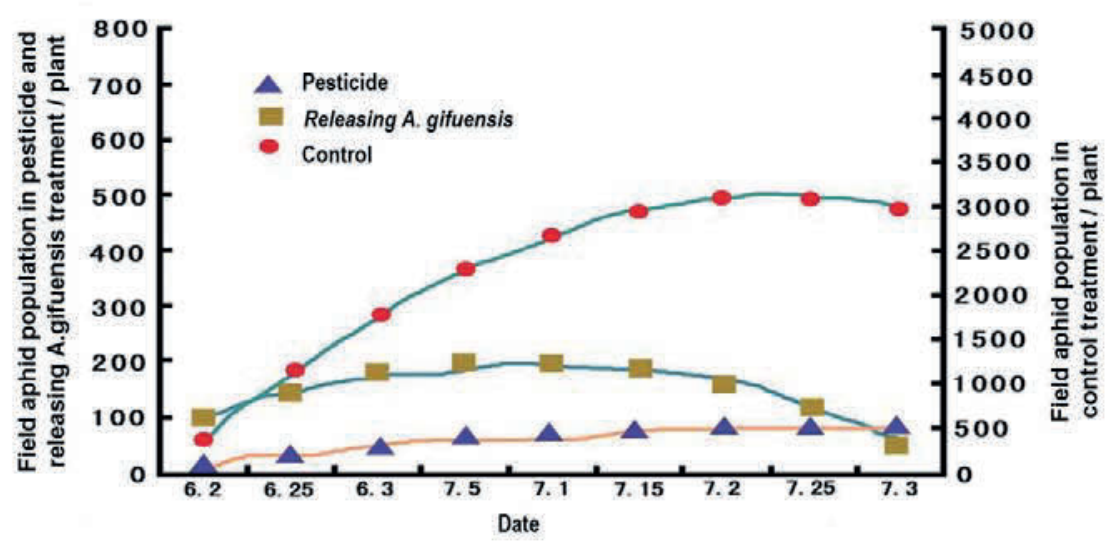

Figure 10. The population dynamics of aphids in tobacco fields treated by insecticides or the release of parasitoids (left y-axis), or not treated (control) (right y-axis), in tobacco fields in Yuxi, China in 2005. 
By 2013, 90\% of all the Yunnan tobacco fields had been covered by the augmentative biological control programme. Starting in 2014, this technology has been applied throughout China. Step by step, this technology has expanded to cover $100 \%$ of the Yunnan tobacco-planting area and $90 \%$ of the Chinese tobacco-planting areas.

Effectiveness of aphid control has reached $80 \%$, better than in pesticide-treated fields ( $\mathrm{Li}$ et al. 2006). The total application area in China has reached 3017547 ha over six years (2010 to 2015), total decrease of pesticide use reached 1966.05 tons, and total decrease of control costs reached USD 230 million. A financial loss of USD 1326 million was avoided and 1 million farm households have benefited from this technology.

The use of $A$. gifuensis against $M$. persicae is the biological control technology that is most widely adopted in China. Aphid population control has been transformed from mainly insecticide- to largely biological control-based, promoting a pest control strategy that changed from passive, reactive and chemical insecticide-based to active, preventive and ecologically-founded, with significant social and environmental benefits.

The biological control of aphids by the mass-release of $A$. gifuensis parasitoids in some other crops (Fig. 11) has also reached high efficacies on a large scale (Shen et al. 2018).

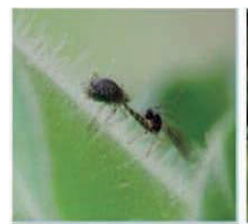

Maize

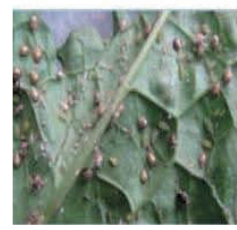

Radish

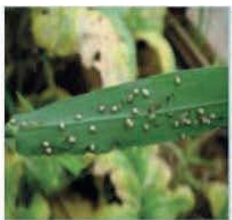

Wheat

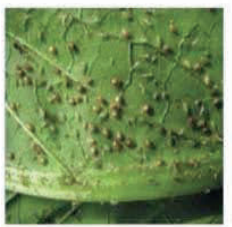

Cabbage

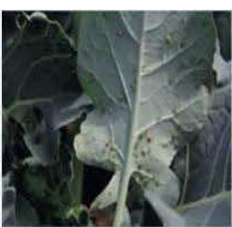

Rape

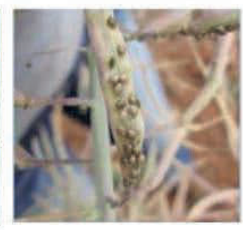

Broccoli

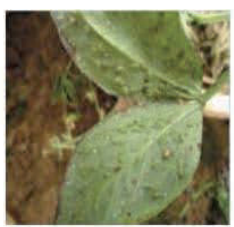

Pea

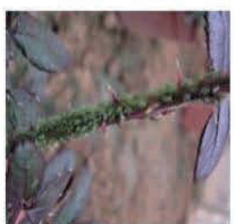

Pepper

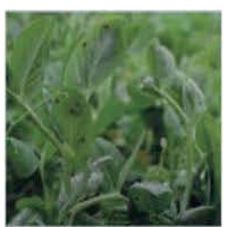

Sweat pea

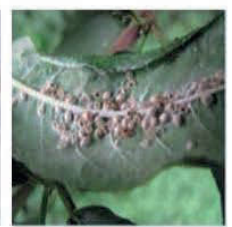

Peach

Figure 11. Examples of biological control of aphids by Aphidius gifuensis augmentative releases on other crops in China.

\section{ACKNOWLEDGEMENTS}

This project has been financially supported by the China Tobacco Company. We thank Professor Zeng-Rong Zhu of Zhejiang University for his critical comments and suggestions for modifications of earlier versions of this chapter. 


\section{REFERENCES}

(AERET) (Association Européenne pour la Recherche et l'Expérimentation Tabacole / European Association for Tobacco Research and Experimentation. 2011. Aphidius gifuensis (Ashmead) safeguards ecological leaf tobacco in Yunnan, China. AERET website, published 18 April 2011.

Bi, Z. B., and Z. D. Ji. 1993. Bionomics of Aphidius gifuensis Ashmead. I. Development stages and morphology of larval stage. Journal of Hebei Agricultural University 16(2): 1-8 [in Chinese].

Chen, J. H. 1979. The fundamental knowledge of taxonomy of Aphidiidae in China. Entomological Knowledge 16: 265-268 [in Chinese].

Chen, S., Y. Liu, and W. Li. 2009. High-density rearing method for Aphidius gifuensis involves inoculating tobacco aphid for twenty days after seeding seedling when seed is small and generating Aphidius gifuensis in fifteen days after inoculation process. Chinese patent: CN101548658-A, 7 October 2009 [in Chinese].

Deng, J. H., X. F. Wu, C. M. Song, J. M. Huang, G. H. Liu, and S. Y. Yang. 2006. Rearing effect of Aphidius gifuensis with nylon-net covered cages in tobacco fields. Journal of Southwest Agricultural University (Natural Science) 28: 66-73 [in Chinese].

Deng, X. G., W. Wu, and S. Yang. 2010. Aphidius gifuensis: Mass rearing and application. First Edition. Environmental Science Press, Beijing, China [in Chinese].

Deng, X., G. Fu, and C. Li. 2011. Large-scale Aphidius gifuensis Ashmead propagating technique for preventing agricultural pest, involves forming mummified aphids after certain number of days, and growing aphids to Aphidius gifuensis in one week. Chinese patent: CN101455190-A; CN101455190-B, 2 November 2011 [in Chinese].

Gao, X. W., B. Z. Zheng, and B. J. Cao. 1992. Resistance in Myzus persicae to organophosphorus and carbamate insecticides in China. Acta Phytophylacica Sinica 19: 365-371 [in Chinese].

Gu, X. H., S. Y. Yang, Y. B. Yu, S. G. Ji, H. L. Yang, J. L. Zhao, L. M. Zhang, and H. G. Zhang. 2015. Application of biological control technology of Myzus persicae with Aphidius gifuensis in Yunnan Province. Chinese Journal of Biological Control 31: 1-7 [in Chinese].

Guan, Q. L., X. C. Shen, and L. F. Yuan. 2016. Agricultural pest resistance and comprehensive control. Agricultural Technology and Equipment 1: 24-25 [in Chinese].

Han, Q. F., P. J. Zhuang, Z. H. Tang, X. Z. Xu, and X. Q. Deng. 1989. The preliminary study on resistance of Myzus persicae to insecticides. Contributions of Shanghai Institute of Entomology 9: 1927 [in Chinese].

He, X. L. 2013. Pest resistance research and summary of governance. World Pesticides 35(5): 34-38 [in Chinese].

Kulash, W. M. 1949. The green peach aphid as a pest of tobacco. Journal of Economic Entomology 42: $677-680$.

Li, M. F., Y. P. Zhang, and X. Z. Wang. 2006. Investigation of the effect to prevent and control aphids by rearing Aphidius gifuensis. Chinese Agricultural Science Bulletin 22: 343-346 [in Chinese].

Liu, J. L., X. D. Shi, G. S. Liu, G. Wang, and Y. H. Yang. 2010. Rules for tobacco intensive seedling production. GB/T 25241. China National Standard Press. 24 pp. [in Chinese].

Lu, H., B. C. Shi, and Z. L. Zhang. 1993. Studies on fecundity of Aphidius gifuensis Ashmead. Acta Agriculturae Boreali-Sinicae 8: 76-79 [in Chinese].

Lu, H., B. C. Shi, Y. Z. Niu, and Z. L. Zhang. 1994. Development thresholds and thermal constants of Aphidius gifuensis and Diaeretiella rapae. Acta Agriculturae Boreali-Sinica 9: 72-75 [in Chinese].

Mackauer, M., and M. J. Way. 1976. Myzus persicae Sulzer, an aphid of world importance, pp. 51-119. In V. L. Delucchi (ed.), Studies in biological control. Cambridge University Press, Cambridge, UK.

Ohta, I., K. Miura, and M. Kobayashi. 2001. Life history parameters during immature stage of Aphidius gifuensis Ashmead (Hymenoptera: Braconidae) on green peach aphid, Myzus persicae (Sulzer) (Homoptera: Aphididae). Applied Entomology and Zoology 36 (1): 103-109.

Shen, S., G. Xu, F. Chen, D. R. Clements, X. Gu, S. Ji, L. Zhang, H. Yang, F. Zhang, K. Yin, H. Zhang, J. Li, and D. Wu. 2018. Effects of Aphidius gifuensis release on insect communities and diversity in tobacco fields of Yunnan Province, China. Pakistan Journal of Biological Sciences 21: 284-291.

Starý, P. 1970. Biology of aphid parasites (Hymenoptera: Aphidiidae) with respect to integrated control. Series Entomologica 6: 1-643.

Wei, J. N., T. F. Li, R. P. Kuang, Z. Wang, T. Yin, X. Wu, L. Zou, W. Z. Zhao, J. Cao, and J. Deng. 2003. Mass rearing of Aphidius gifuensis (Hymenoptera: Aphidiidae) for biological control of Myzus persicae (Homoptera: Aphididae). Biocontrol Science and Technology 13: 87-97. 
Wei, J. N., B. B. Bai, T. S. Yin, Y. Wang, Y. Yang, L. H. Zhao, R. P. Kuang, and R. J. Xiang. 2005. Development and use of parasitoids (Hymenoptera: Aphidiidae \& Aphelinidae) for biological control of aphids in China. Biocontrol Science and Technology 15: 533-551.

Wu, X. F., T. F. Li, and J. N. Wei. 2000.Temperature effects on development and fecundity of Aphidius gifuensis Ashmead. Zoological Research 21(3): 192-198 [in Chinese].

Yang, S., S. Y. Yang, C. P. Zhang, J. N. Wei, and R. P. Kuang. 2009. Population dynamics of Myzus persicae on tobacco in Yunnan Province, China, before and after augmentative releases of Aphidius gifuensis. Biocontrol Science and Technology 19(2): 219-228.

Yang, S., S. Y. Yang, C. P. Zhang, and R.P. Kuang. 2010. Changes of population dynamics of Myzus persicae and effects of its natural enemies in tobacco fields. Journal of Southwest China Normal University. Natural Science 35: 68-72 [in Chinese].

Yang, S., J. N. Wei, S. Y. Yang, and R. P. Kuang. 2011. Current status and future trends of augmentative release of Aphidius gifuensis for control of Myzus persicae in China's Yunnan Province. Journal Entomological Research Society 13: 87-99.

Yang, S. Y., and J. L. Zhao. 2009. Mass rearing and release technique of Aphidius gifuensis, pp. 186-191. In Entomology Annual 2009 Conference in Yunnan Province, China [in Chinese].

Yunnan Tobacco Yuxi City Company. 2010. Aphidius gifuensis mass rearing and application. China Environmental Science Press. ISBN: 7511102441, 9787511102447, China [in Chinese].

Yun, D., T. L. Gao, B. J. Yu, S. Y. Yang, J. Zhu, and Z. H. Tian. 2012. Technical specification for Myzus persicae biological control with Aphidius gifuensis. YC/T 437-2012. China National Standard Press. 12 pp. [in Chinese].

Zhao, W. Y., C. P. Din, and W. L. Zhang. 1980. The bionomics of Aphidius gifuensis Ashmead and its utilization for the control of tobacco aphid Myzus persicae Sulzer. Zoological Research 1: 405-416 [in Chinese]. 